\title{
The Coma Cluster at $\gamma$-ray energies: Multifrequency constraints
}

\author{
A. Reimer ${ }^{1}$, O. Reimer ${ }^{1}$, R. Schlickeiser ${ }^{1}$, and A. Iyudin ${ }^{2}$ \\ ${ }^{1}$ Institut für Theoretische Physik IV, Ruhr-Universität Bochum, 44780 Bochum, Germany \\ e-mail: afm@tp4.rub.de \\ ${ }^{2}$ Max-Planck Institut für extraterrestrische Physik, Gießenbachstraße, 85740 Garching, Germany
}

Received 22 March 2004 / Accepted 1 June 2004

\begin{abstract}
The Coma cluster exhibits evidence of a high-energy non-thermal particle population. At frequencies $>1 \mathrm{GHz}$ recent radio halo observations confirm a significant spectral steepening of the volume-integrated emission. We calculate the volumeaveraged high-energy spectrum due to inverse Compton scattering off the CMB radiation field and non-thermal bremsstrahlung according to an exponential cutoff in the electron spectrum as deduced from the radio observations. The synchrotron radiation from secondary pairs, created from the decay of charged mesons produced in hadronic $p p$-interactions, is found to set significant constraints on the energy content of relativistic hadrons in Coma. This limits the maximum flux at high energies. Our findings support a low ratio of relativistic hadron to thermal energy density. Predictions for Coma's high energy emission are discussed in the light of current and expected abilities of upcoming $\gamma$-ray instruments.
\end{abstract}

Key words. galaxies: clusters: individual: Coma - gamma rays: theory - radiation mechanisms: non-thermal

\section{Introduction}

Clusters of galaxies are conglomerates of a large number of galaxies that are gravitationally bound and confine a large fraction of the mass in the universe. One of the controversially discussed properties of clusters of galaxies are their nonthermal components which include cosmic rays as well as turbulence and non-regular magnetic fields. The non-thermal pressure has an important impact on the evolution of galaxy clusters. Reaching a better understanding of these components is mainly driven by the importance of the non-thermal pressure in the evolution of galaxy clusters. In this paper we discuss only cosmic rays as the most easily testable of all non-thermal components. Several mechanisms have been proposed that lead to relativistic particles in the intracluster medium (ICM), e.g. particle acceleration during their formation and evolution, in merger shocks (Takizawa \& Naito 2000; Gabici \& Blasi 2003b; Berrington \& Dermer 2003; Miniati et al. 2001a), accretion shocks (Colafrancesco \& Blasi 1998), intergalactic termination shocks from the winds of the galaxies (Völk et al. 1996), or reacceleration of injected mildly relativistic particles from powerful cluster members (Enßlin et al. 1997). Indeed, the detection of synchrotron radiation from cluster radio halos and relics signals the existence of relativistic electrons $\left(\mathrm{e}^{-}\right)$in the intracluster medium (ICM). The most prominent cluster that possesses a radio halo is the Coma cluster (Abell 1656), located at redshift $z=0.0232$ (Struble \& Rood 1991) (corresponding to $\sim 90 \mathrm{Mpc}$ for $H_{0}=75 \mathrm{~km} \mathrm{~s}^{-1} \mathrm{Mpc}^{-1}$ ). At frequencies below $\sim 1 \mathrm{GHz}$, observed volume-integrated fluxes are satisfactorily fitted by a pure power law. Observations at higher frequencies gave evidence that a significant spectral steepening of the integrated emission occurs in Coma's radio halo (Schlickeiser et al. 1987), recently confirmed by Thierbach et al. (2003; TH03). A hard X-ray (HXR) excess has been detected by the Rossi X-ray Timing Explorer (RXTE) (Rephaeli et al. 1999) and BeppoSAX (Fusco-Femiano et al. 2004). Coma is one of the few clusters where an EUV excess is conclusively established (Bowyer \& Berghöfer 1998; Lieu et al. 1999). The spread of the soft X-ray to EUV emission is, however, still debated (Kaastra et al. 2003), and may possibly extend up to the turnaround radius. Here we restrict our considerations to the size of the radio halo $\left(\sim 10^{\prime} \times 30^{\prime}\right.$; TH03). By comparing the radio synchrotron spectrum with this excess radiation, interpreted as Inverse Compton (IC) scattering off photons from the cosmic microwave background (CMB) by the same $\mathrm{e}^{-}$population, volume-averaged magnetic fields of $B=0.1 \ldots 0.3 \mu \mathrm{G}$ have been deduced (Fusco-Femiano et al. 2004; Rephaeli et al. 1999). Faraday rotation measurements gave $B \sim 2 \ldots 10 \mu \mathrm{G}$ (Kim et al. 1990; Clarke et al. 2001).

Many models for non-thermal radiation from the Coma cluster predict significant emission at $\geq 100 \mathrm{MeV}$ due to relativistic $\mathrm{e}^{-}$and ions (Sarazin 1999a; Atoyan \& Völk 2000; Miniati 2003; Gabici \& Blasi 2004). They often assume a power law to $\sim 10^{6.7} \mathrm{MeV}$ for the $\mathrm{e}^{-}$population responsible for the dominating synchrotron component. Here we investigate the consequences of the decline in the $\mathrm{e}^{-}$spectrum at $\sim 10^{4} \mathrm{MeV}$ as deduced from recent radio observations of Coma $\mathrm{C}$ for the expected high energy flux. In Sect. 2 the IC and non-thermal bremsstrahlung spectrum from the steepening $\mathrm{e}^{-}$distribution is calculated. In Sect. 3 we derive limits imposed 


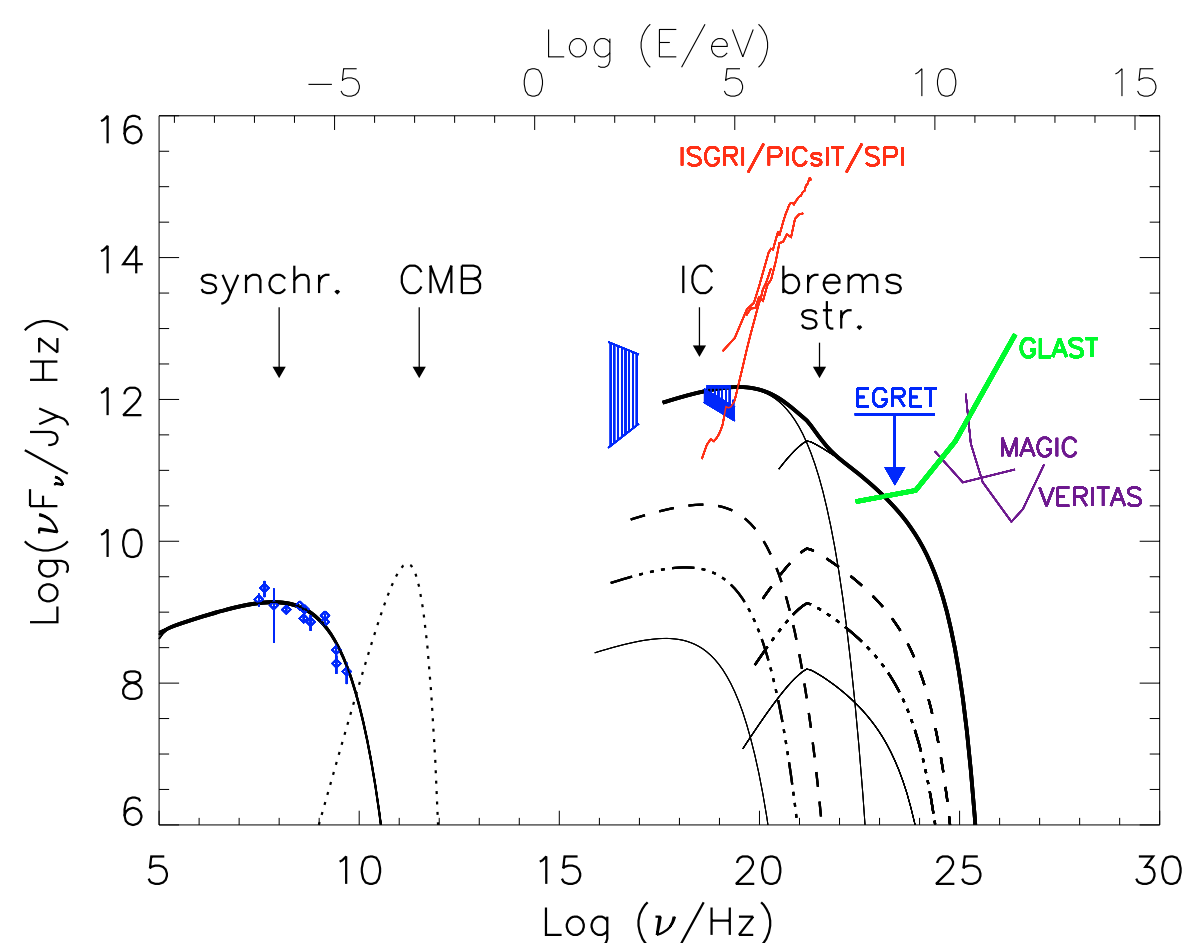

Fig. 1. Broad band continuum spectrum of Coma. The radio data and the best-fit spectrum at source (corrected for self-absorption) are taken from TH03 (not corrected for the thermal Sunyaev-Zeldovich effect). The dotted line represents the CMB field corrected for the thermal Sunyaev-Zeldovich effect using a y-parameter of $0.75 \times 10^{-4}$ (Enßlin 2002). The IC and non-thermal bremsstrahlung fluxes are calculated for field strengths $B=0.1,0.68,1.9,6 \mu \mathrm{G}$ (from upper to lower curves) using an exponential $\mathrm{e}^{-}$distribution (with adjusted $Q_{0}, p_{c}$ to fit the radio data), and $n_{i}=10^{-3} \mathrm{~cm}^{-3}$. They are extended to lower energies assuming the synchrotron spectrum follows a power law down to at least $10^{-9} \mathrm{eV}$. The hatched regions in the X-ray domain represent the data from PDS/BeppoSAX (Fusco-Femiano et al. 2004), HEXTE/RXTE (Rephaeli et al. 1999) and EUVE (Lieu et al. 1999). The RXTE and EUVE fluxes are integrated within a ring of $21^{\prime}$ and $18^{\prime}$, respectively, while the PDS data include only fluxes within $8^{\prime}$ from the cluster center.

by the broadband observations for the $\pi^{0}$-decay $\gamma$-ray component including its secondary pair initiated radiation. Finally we discuss Coma's detectability with current/future $\gamma$-ray instruments such as INTEGRAL, GLAST-LAT and the new Imaging Air Cherenkov Telescopes (IACTS).

\section{Non-thermal electron spectrum and radiation}

The volume-integrated radio emission from the radio halo has been studied in detail by e.g. Schlickeiser et al. (1987), Kim et al. (1990), Venturi et al. (1990), Giovannini et al. (1993), Deiss et al. (1997), TH03. Figure 1 shows the volumeintegrated radio continuum spectrum of the diffuse radio halo source Coma $\mathrm{C}$ as published in TH03 with the best fit model. TH03 confirmed the findings of Schlickeiser et al. (1987) that among the three basic models for cluster halos (primary electron model: Jaffe (1977), Rephaeli et al. (1999); secondary electron model: e.g. Dennison (1980); in-situ acceleration model: Jaffe (1977), Roland (1981), Schlickeiser et al. (1987)) the in-situ acceleration model fits the observed exponential steepening of the synchrotron spectrum best. This model, though discussed critically by Petrosian (2001), considers shock wave and resonant diffusion acceleration out of a thermal pool of particles where radiation losses and particle escape have been taken into account. A secondary origin for the radio halo has been proposed by many authors (e.g. Dennison 1980; Blasi \& Colafrancesco 1999; Dolag \& Enßlin 2000; Atoyan \& Völk 2000; Blasi 2001; Miniati et al. 2001b). Recently, however, arguments have been given which suggest that secondary pairs as the underlying particle population of the radio halo emission are problematic (Brunetti 2003; Kuo et al. 2004). Along these Brunetti et al. (2004) found that the observations of non-thermal radiation of galaxy clusters are only reproducible within the picture of particle acceleration through cluster merger generated Alvén waves, if the fraction of relativistic hadrons in the ICM is small (5-10\%). This hadron content is insufficient to reproduce the radio halo from secondary pairs (see below). Curved spectra are also possible at an energy where losses balance the acceleration rate if the acceleration time decreases more slowly than the loss time. In the following we therefore consider an exponential shape of the $\mathrm{e}^{-}$spectral distribution, suitable to explain the volumeaveraged synchrotron spectrum, irrespective of its formation mechanism. This rather phenomenological ansatz will not shed light on the mechanisms responsible for the formation of the $\mathrm{e}^{-}$distribution, however, it leads to model-independent constraints for the high-energy component arising from this leptonic particle population.

We fit the radio flux density with a power law synchrotron spectrum extended by an exponential cutoff:

$I_{\text {syn }}(v) \propto v^{(3-s) / 2} \exp \left(-\sqrt{v / v_{s}}\right)$ 
with $s=4.6$ and $v_{s}=0.44 \mathrm{GHz}$ (TH03). Synchrotronself absorption will affect the synchrotron spectrum at low energies. For an estimated path length of $\sim 280 \mathrm{kpc}$ through the cluster (TH03) we found the turnover frequencies at $\sim$ a few $100 \mathrm{kHz}$ (see Fig. 1). Below $\sim 0.2 \mathrm{MHz}$ free-free absorption in the disk of the Milky Way suppresses the radio intensity from Coma observed at Earth. In addition, the Razin-Tsytovich effect causes the radio spectrum to decline rapidly to low frequencies from $v_{R} \lesssim 20\left(n_{\mathrm{e}} / B_{\mathrm{G}}\right) \mathrm{Hz}\left(n_{\mathrm{e}}\right.$ is the $\mathrm{e}^{-}$density in $\mathrm{cm}^{-3}$, and $B_{\mathrm{G}}$ is the magnetic field in Gauss) with a turning point between $0.01 \ldots 0.6 \mathrm{MHz}$ for the expected field strengths in Coma. Turnovers below MHz-frequencies are, however, not detectable with ground-based radio observatories due to ionospheric effects, and have to await future space-based low-frequency observatories.

For a given magnetic field $B$ the corresponding volumeintegrated $\mathrm{e}^{-}$spectrum

$\eta(p)=Q_{0} p^{-s} \exp \left(-p / p_{c}\right)$

(Schlickeiser et al. 1987) with normalization $Q_{0}$ and cutoff $\mathrm{e}^{-}$momentum $p_{c}$ can then be determined. Values for $p_{c}$ considering magnetic field strengths of $0.1 \ldots 6 \mu \mathrm{G}$ lie from $2153 \ldots 16667 \mathrm{MeV} / c$. IC scattering off $\mathrm{CMB}$ photons with photon energies $\bar{\epsilon}_{\mathrm{CMB}} \simeq 6 \times 10^{-4} \mathrm{eV}\left(T_{\mathrm{CMB}}=2.7 \mathrm{~K}\right)$ by these $\mathrm{e}^{-}$is therefore restricted to the Thomson regime. In the $\delta$-approximation the intensity of the IC scattered radiation as a function of photon energy $E_{\gamma}$ can be analytically expressed if the target photon density distribution $n(\epsilon)$ is sufficiently peaked so that $E_{c}=\left(p_{c} / m_{\mathrm{e}} c\right)^{2} \epsilon \approx\left(p_{c} / m_{\mathrm{e}} c\right)^{2} \bar{\epsilon}$ :

$$
\begin{aligned}
I_{C}\left(E_{\gamma}\right)= & \frac{c \sigma_{T} E_{\gamma}}{4 \pi d_{L}^{2}} \int_{0}^{\infty} \mathrm{d} \epsilon n(\epsilon) \int_{p_{\min }}^{\infty} \mathrm{d} p p^{2} \eta(p) \delta\left(E_{\gamma}-\left(\frac{p}{m_{\mathrm{e}} c}\right)^{2} \epsilon\right) \\
= & \frac{c \sigma_{T} Q_{0}}{8 \pi d_{L}^{2}}\left(k T_{\mathrm{CMB}}\right)^{(3+s) / 2} \Gamma\left(\frac{s+3}{2}\right) \xi\left(\frac{s+3}{2}\right) \\
& \times\left(m_{\mathrm{e}} c\right)^{3-s} E_{\gamma}^{(3-s) / 2} \exp \left(-\sqrt{E_{\gamma} / E_{c}}\right)
\end{aligned}
$$

with $\Gamma$ the Gamma function, $\xi$ is Riemann's zeta function, $m_{\mathrm{e}} c^{2}$ the $\mathrm{e}^{-}$rest mass, $\sigma_{T}=6.65 \times 10^{-25} \mathrm{~cm}^{2}, d_{L}$ Coma's luminosity distance and $n(\epsilon)$ is the CMB photon density. Figure 1 shows the resulting IC spectra for an equipartition magnetic field ranging between $0.68 \ldots 1.9 \mu \mathrm{G}$ (TH03), for the central magnetic field in Coma $C(B \sim 6 \mu \mathrm{G}$ : Feretti et al. 1995) and for $B=0.1 \mu \mathrm{G}$ appropriate to explain the HXR excess emission.

The non-thermal volume-averaged bremsstrahlung intensity using the primordial ${ }^{4} \mathrm{He}$ mass fraction of 0.24

$$
I_{B}\left(E_{\gamma}\right)=\frac{1.18 n_{i} c E_{\gamma}}{4 \pi d_{L}^{2}} \int_{\max \left(E_{\gamma} m_{\mathrm{e}} c, p_{\min }\right)}^{p_{\max }} \mathrm{d} p p^{2} \eta(p) \frac{\mathrm{d} \sigma}{\mathrm{d} E_{\gamma}}
$$

is calculated in the relativistic limit using the differential cross section from Blumenthal (1970). For a mean gas density $n_{i} \sim 10^{-3} \mathrm{~cm}^{-3}$ in Coma we find the compound IC and bremsstrahlung spectrum from the observed synchrotronemitting $\mathrm{e}^{-}$distribution always below the EGRET upper limit (Reimer et al. 2003). The steepening of the $\mathrm{e}^{-}$spectrum at $10^{3 \ldots 4} \mathrm{MeV}$ causes the IC component to decline at $\sim 1-10 \mathrm{MeV}$; non-thermal bremsstrahlung dominates until its decline at a few $\mathrm{GeV}$. This is in contrast to works where the primary $\mathrm{e}^{-}$spectrum extends to several $10^{7} \mathrm{MeV}$ (e.g. Atoyan $\&$ Völk 2000; Miniati 2003). Here the volume-averaged IC and bremsstrahlung spectra extend to $\mathrm{GeV}-\mathrm{TeV}$ energies and may dominate the $\gamma$-ray domain, depending on the strength of the $\pi^{0}$-decay component.

\section{Hadronic cosmic ray - gas interactions}

Interactions between cosmic ray protons and nucleons of the ICM gas component are very rare and occur on average once in a Hubble time in Coma. The number of collisions is usually time-dependent, in particular higher than average soon after a (e.g. merger) shock has started to develop, due to newly injected particles. In the case of a high cosmic ray hadron content $\gamma$-rays from the decay of $\pi^{0}$ are expected to determine the energy range $>1 \mathrm{GeV}$. Additionally, radiation from the secondary pairs, generated through the decay of charged mesons that are produced by hadronic $p p$-collisions, is expected to contribute to the overall broad band spectrum. The short cooling time scales of those pairs radiating in the $\mathrm{GHz}$ and hard X-ray to $\gamma$-ray band leads to quasi-stationary pair populations at these energies on a very short time scale. As a consequence a direct relation between the $\pi^{0}$-decay $\gamma$-ray spectrum and the radiation spectrum from the (high energy) secondary pairs is expected.

The spectral index of Coma's putative relativistic proton distribution and its normalization has not yet been determined observationally. Nevertheless some plausible arguments can be found to limit the parameter space. Because cosmic ray protons are stored efficiently in galaxy clusters for cosmological times (Völk et al. 1996; Berezinsky et al. 1997), the radiation from the secondary pairs reflects the injected proton spectrum, and the global proton spectrum should be not significantly different from the injected one if uniform injection throughout the cluster is assumed. The structure formation shock scenario gives injection spectral indices of $\alpha_{\mathrm{p}}=2.0 \ldots 2.5$ for strong shocks (Miniati 2003). For merger shocks plunging into the cluster body from the periphery $\alpha_{\mathrm{p}}$ can evolve from $2 \ldots 5$ (Berrington \& Dermer 2003). The normalization of the proton component is limited by three constraints: Firstly, the $\pi^{0}$-decay $\gamma$-rays must not be overproduced to violate the EGRET upper limit. Secondly, IC scattering off CMB photons by the secondary pair $\left(\mathrm{e}^{ \pm}\right)$population produced in $p p$-interactions leads to a further radiation component that covers the energy range from $\sim 5 \mathrm{eV}$ (corresponding to $\mathrm{e}^{ \pm}$of energy $\sim 50 \mathrm{MeV}$ ) to a few GeV. This component is constrained by the HXR flux and EGRET upper limit. The expected non-thermal bremsstrahlung from these secondary $\mathrm{e}^{ \pm}$lies always below the corresponding IC flux level. Lastly, these secondary $\mathrm{e}^{ \pm}$also emit synchrotron photons, and this leads to a constraint imposed by the radio observations. Figure 2 shows the resulting stationary $\gamma$-ray spectra for $\alpha_{\mathrm{p}}=2.1,2.3$ and 2.5, calculated using the formalism given in Pfrommer \& Enßlin (2004) for the $\pi^{0}$-decay $\gamma$-ray production and secondary pair production. We limited the proton spectrum, assumed to be uniformly injected throughout the cluster, to $10^{6} \mathrm{GeV}$ since higher energetic protons are difficult to confine within the cluster size (Colafrancesco \& Blasi 1998). 


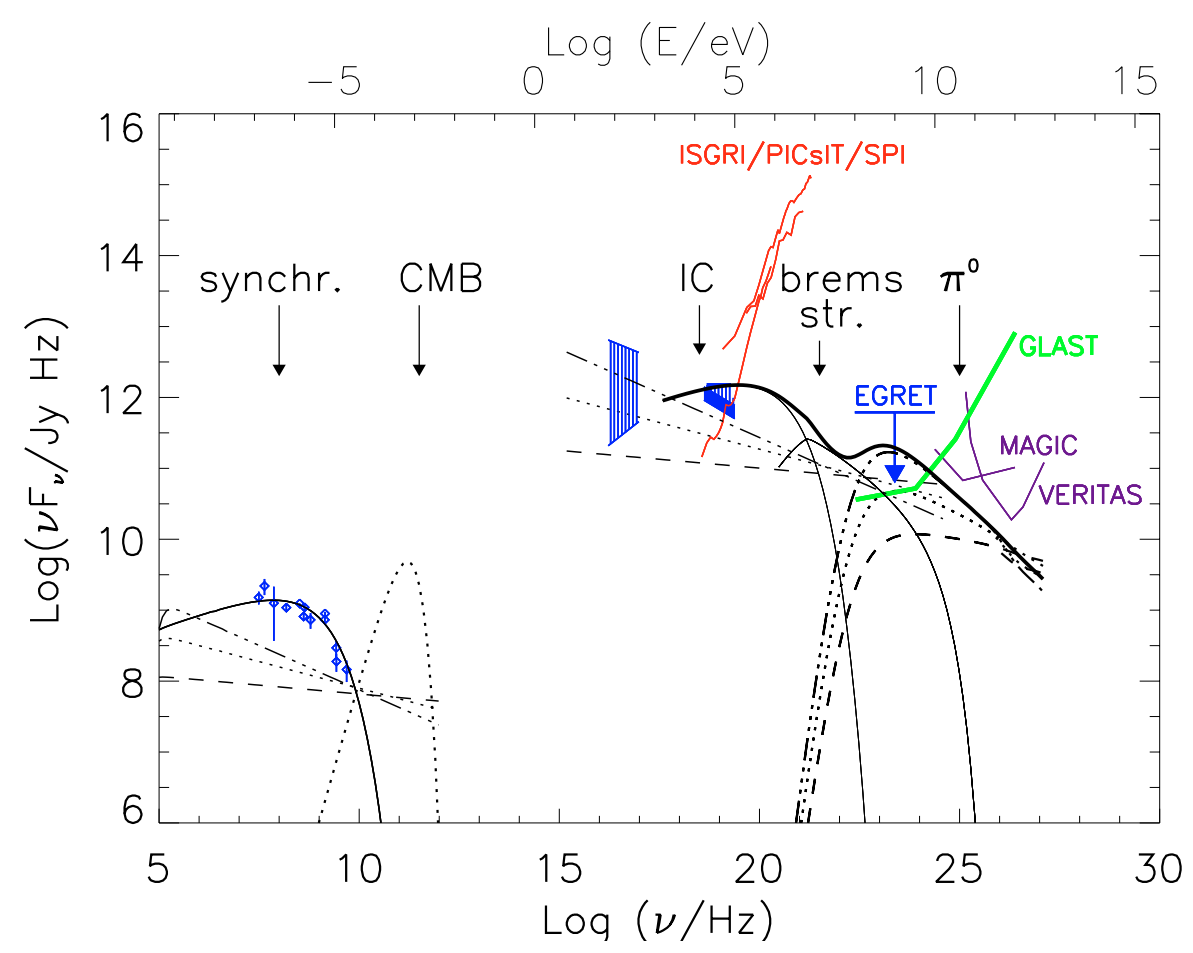

Fig. 2. Same as Fig. 1 but the IC and non-thermal bremsstrahlung fluxes are shown only for a field strength $B=0.1 \mu \mathrm{G}$. The $\pi^{0}$-decay $\gamma$-ray spectra (most right curves) are calculated for a $\alpha_{\mathrm{p}}=2.1$ (dashed line), 2.3 (dotted line), 2.5 (dashed-dotted line) proton spectrum and the normalization of the particle spectra are adjusted to avoid violating the EGRET upper limit as well as the integral fluxes in the HXR and radio domain (see text). The required relativistic proton energy densities are 3\%,8\% and 28\% of the thermal energy content for $\alpha_{\mathrm{p}}=2.1,2.3$ and 2.5 , respectively. The corresponding IC and synchrotron fluxes are shown as dashed/dotted lines.

The use of gas and proton density profiles as applied in e.g. Blasi (1999) instead of the volume-averaged parameters leads to only minor changes in the $\pi^{0}$-decay $\gamma$-ray intensity for the volume of Coma considered here (with an effective radius of $\sim 330 \mathrm{kpc}$ ). Above $\sim 1 \mathrm{TeV}$, photon absorption due to photonphoton pair production in the cosmic infrared-to-optical background radiation field must be taken into account. For this correction we used the background models in Aharonian (2001).

Proton energy densities $u_{\mathrm{p}}$ are calculated in the following from the proton spectrum above the threshold for hadronic $p p$-collisions, and are compared to Coma's thermal energy density $u_{\text {therm }} \approx 3.8 \times 10^{-11} \mathrm{erg} / \mathrm{cm}^{-3}$ (for $k T_{\mathrm{e}}=8.2 \mathrm{keV}$, a thermal $\mathrm{e}^{-}$density of $10^{-3} \mathrm{~cm}^{-3}$ and a ${ }^{4} \mathrm{He}$ mass fraction of 0.24$)$. The synchrotron flux in the $\mathrm{MHz}$-to- $\mathrm{GHz}$ regime from the secondary pairs is dependent on $u_{\mathrm{p}}$ and $\alpha_{\mathrm{p}}$ as well as on $B$.

For $\alpha_{\mathrm{p}} \sim 2.4$ and $X_{\mathrm{p}} \equiv u_{\mathrm{p}} / u_{\text {therm }} \sim 20 \%$ the radio data are explainable by synchrotron emission from secondary $\mathrm{e}^{ \pm}$in a volume-averaged magnetic field of $0.15 \mu \mathrm{G}$ if the steepening of the radio spectrum at high frequencies is disregarded, in agreement with Blasi \& Colafrancesco (1999), Dolag \& Enßlin (2000). If the steepening of the $>1 \mathrm{GHz}$ radio data is taken into account, obviously the synchrotron flux from the secondary pairs must lie below the GHz-radio observations. In fact, we find that these high frequency radio data place the most stringent constraint on the proton energy content in the Coma Cluster. The resulting upper limits for the relativistic hadronic energy density of $X_{\mathrm{p}}<3 \% \ldots 0.009 \%, X_{\mathrm{p}}<8 \% \ldots 0.01 \%$ and $X_{\mathrm{p}}<28 \% \ldots 0.07 \%$ (assuming $B=0.1 \ldots 2 \mu \mathrm{G}$ ) for $\alpha_{\mathrm{p}}=2.1$,
2.3 and 2.5, respectively, are significantly lower than those used in structure formation triggered acceleration scenarios. For example, the model of Miniati (2003) required $\sim 34 \%$ of the thermal energy in the form of cosmic ray ions for $B=0.15 \mu \mathrm{G}$, and $\sim 4 \%$ for $B=0.5 \mu \mathrm{G}$ with a proton spectrum $\alpha_{\mathrm{p}} \sim 2$ to explain the radio halo emission as originating from the secondary pairs. Our cosmic ray limits are also lower than the limits derived from Pfrommer \& Enßlin (2004) $\left(X_{\mathrm{p}}<45 \% \ldots 25 \%\right.$ for $\left.\alpha_{\mathrm{p}}=2.1 \ldots 2.5\right)$ which solely relied on the EGRET upper limit constraint. For the case $\alpha_{\mathrm{p}}=2.1$ and $B=0.68 \mu \mathrm{G}$ we find approximative equipartition between particles and fields with $X_{\mathrm{p}} \approx 0.05 \%$. Except for proton spectra harder than $\alpha_{\mathrm{p}} \leq 2.3$ we find in all cases the radiation spectra at $>1 \mathrm{keV}$ from the secondary pairs to lie below the corresponding photon spectra from the primaries. This is shown in Fig. 2 for $B=0.1 \mu \mathrm{G}$, which simultanously gives the most optimistic flux predictions at high energies. Below the hard X-ray band, IC from both primaries and secondary pairs determine the shape of the volumeaveraged spectrum. Depending on the proton spectral index and overall hadron content in Coma, a turnover from primaries' to secondaries' dominated IC below the soft X-ray band may occur. This is in agreement with the finding of Bowyer \& Berghöfer (1998) that the non-thermal halo component detected with the EUVE may stem from an additional component of low-energy cosmic ray $\mathrm{e}^{-}$which we interpret as the secondary pairs. Independent hints for a EUV emission of secondary pair origin has been given by Bowyer et al. (2004) who found a striking spatial correlation between the EUVE excess 
and ROSAT thermal hard X-ray flux based on a re-examination of the EUVE data. So far the EUVE excess radiation, if considered to be of non-thermal origin, has been interpreted either as IC emission from low energy relic $\mathrm{e}^{-}$(Sarazin 1999a; Atoyan \& Völk 2000) or explained by a spectral break between the EUVE and HXR radiating $\mathrm{e}^{-}$(induced by a certain particle injection scenario), while a secondary pair origin had erroneously been ruled out (see Bowyer et al. (2004) for a discussion).

In the GLAST energy range non-thermal bremsstrahlung, followed by $\pi^{0}$-decay $\gamma$-rays above $\sim 0.1 \mathrm{GeV}$, will dominate, similar to the predictions given in Sarazin (1999b). Only for hard input proton spectra will IC radiation from the secondary pairs determine the $\mathrm{GeV}$ radiation. No $\gamma$-ray emission above $\sim 10 \mathrm{GeV}$ is expected for proton injection spectra as steep as $\alpha_{\mathrm{p}}=5$.

\section{Detectability with gamma ray instruments}

Advances in the spatial and spectral resolving capabilities of current/future high energy instruments will allow studies of the Coma cluster continuum emission at energies higher than the hard X-rays. A detection in $\gamma$-rays may help clarify on the spectral extent of the cluster's non-thermal emission, may provide constraints on the acceleration processes realized in Coma, and also yield more precise estimates than currently possible of the galaxy cluster contribution to the extragalactic $\gamma$-ray background (Berrington \& Dermer 2003; Fujita et al. 2003; Gabici \& Blasi 2003a; Miniati 2002). Observationally upper limits are currently provided by OSSE (Rephaeli et al. 1994) and EGRET (Reimer et al. 2003). INTEGRAL with its moderate continuum sensitivity ${ }^{1}$ permits a chance to detect Coma as a marginally extended source up to a few $100 \mathrm{keV}$ by ISGRI (Goldoni et al. 2001). PICsIT and SPI, however, will not be able to detect the Coma cluster given realistic observation times of $<10^{7} \mathrm{~s}$. The current generation of imaging Cherenkov telescopes (IACTS; Weekes et al. 2002), in particular in northern locations, will reach the required sensitivity only if significantly more than $50 \mathrm{~h}$ of observation will be accumulated. Even then, the excellent resolving capabilities of IACTs cannot be used to its full advantage due to the extended character of Coma's emission where IACTs have a reduced sensitivity. This applies in particular to cases where the dominant sub-GeV/TeV-emission component originates from the outskirts, e.g., due to accretion shocks (Gabici \& Blasi 2004).

AGILE $^{2}$, expected to have a similar performance to EGRET, might be able to verify the EGRET upper limit. It is the Large Area Telescope (LAT) ${ }^{3}$, the main instrument aboard GLAST, that has a realistic chance to finally detect Coma in continuum $\gamma$-rays. With its significantly better spectral and spatial resolution, and up two orders of magnitude improved

\footnotetext{
${ }^{1}$ http://www.rssd.esa.int/Integral/A02/

${ }^{2}$ http://agile.mi.iasf.cnr.it/Homepage/ performances.shtml

${ }^{3}$ http://www-glast.slac.stanford.edu/software/IS/ glast_lat_performance.htm
}

sensitivity compared to EGRET, the $\pi^{0}$-decay component will be within the reach of LAT. Due to the similar spatial extent of Coma C and LAT's point spread function at GeV energies, spatially resolved spectral information is difficult to gain. Although photon-limited, LAT will benefit from its wide field-of-view, that allows a steady accumulation of exposure throughout the expected mission life time for any observable object in the sky, including the Coma cluster.

\section{Conclusions}

The present work considers the role of the recently confirmed steepening of Coma's radio halo spectrum in the $\mathrm{GHz}$ band for predicted fluxes in the high energy regime. Indeed, we found that the steepening radio spectrum efficiently constrains the amount of hadronic cosmic rays through the radiation channel of the secondary pairs produced in the decay chain of the hadronically produced charged mesons. The implied upper limits for the hadronic cosmic ray energy density range from $0.01 \% \ldots 28 \%$ of the thermal energy density, depending on the magnetic field $(B=0.1 \mu \mathrm{G} \ldots 2 \mu \mathrm{G})$ and proton injection spectral index $\left(\alpha_{\mathrm{p}}=2.1 \ldots 2.5\right)$, and are smaller than those used by other works. This might have severe implications for the evolution of galaxy clusters, acceleration scenarios in cluster of galaxies and the origin (secondary versus primary electron scenario) of Coma's radio halo.

Below the soft X-ray band we found that a turnover from primaries' to secondaries' dominated IC emission may occur, depending on $\alpha_{\mathrm{p}}$ and the hadronic cosmic ray content in Coma. This is in agreement with the suggestions of Bowyer \& Berghöfer (1998) that the non-thermal halo component detected with the EUVE may stem from an additional population of low-energy cosmic ray electrons which could in this scenario be interpreted as the secondary pair component. Independent hints for a EUV emission of secondary pair origin has been given by Bowyer et al. (2004) on the basis of a spatial correlation analysis between the EUVE excess and the ROSAT thermal hard X-ray flux.

The steepening of the $\mathrm{GHz}$ radio spectrum leads to a decline of the IC and bremsstrahlung component of the $\gamma$-ray spectrum already at $1-10 \mathrm{MeV}$ and a few $\mathrm{GeV}$, respectively, depending on the magnetic field. We have shown that the current continuum sensitivity of INTEGRAL's ISGRI at $>$ a few $100 \mathrm{keV}$ for a $10^{6} \mathrm{~s}$ observation is insufficient to detect even the most optimistic predicted flux from Coma. The situation is even worse for PICsIT and SPI.

$\pi^{0}$-decay $\gamma$-rays may extend Coma's $\gamma$-ray spectrum to $\mathrm{TeV}$ energies. However, significant limits to its absolute flux are imposed by the radio spectrum (see above). This leads to flux limits that are below the point source minimum flux after $50 \mathrm{~h}$ on-source observations reached by modern generation northern hemisphere Cherenkov telescopes like MAGIC and VERITAS. The case is even worse for extended sources.

All predictions presented here are based on the assumption of power-law proton spectra in the Coma cluster. Curved proton spectra may be possible as a result of re-acceleration of the confined cosmic ray hadrons in clusters of galaxies 
(Gabici \& Blasi 2003b), and this might lead to corresponding changes in the predicted limits.

It will be LAT of the GLAST mission that might finally be able to detect Coma in the $\gamma$-ray band if the magnetic field and/or Coma's hadronic energy content is favorable.

Acknowledgements. AR's research is funded by DESY-HS, project 05CH1PCA/6, OR's by DLR QV0002. We thank the referee, P. Blasi, for his constructive comments.

\section{References}

Aharonian, F. A. 2001, Proc. 27th ICRC, Hamburg, 8, 250 Atoyan, A. M., \& Völk, H. J. 2000, ApJ, 535, 45

Berezinsky, V. S., Blasi, P., \& Ptuskin, V. S. 1997, ApJ, 487, 529

Berrington, R. C., \& Dermer, C. D. 2003, ApJ, 594, 709

Blasi, P., \& Colafrancesco, S. 1999, Astropart. Phys., 122, 169

Blasi, P. 1999, ApJ, 525, 603

Blasi, P. 2001, Astropart. Phys., 15, 223

Blumenthal, G. R., \& Gould, R. J. 1970, Rev. Mod. Phys., 42, 237

Bowyer, S., \& Berghöfer, T. W. 1998, ApJ, 505, 502

Bowyer, S., Korpela, E. J., Lampton, M., \& Jones, T. W. 2004, ApJ, 605, 168

Brunetti, G. 2003, in Matter and Energy in Clusters of Galaxies, ed. S.

Bowyer, \& C.-Y. Hwang, San Francisco, ASP Conf. Ser., 301, 349

Brunetti, G., et al. 2004, MNRAS, in press

Colafrancesco, S., \& Blasi, P. 1998, Astropart. Phys., 9, 227

Clarke, T. E., Kronberg, P., \& Böhringer, H. 2001, ApJ, 547, L111

Deiss, B. M., Reich, W., Lesch, H., et al. 1997, A\&A, 321, 55

Dennison, B. 1980, ApJ, 239, L93

Dolag, K., \& Enßlin, T. A. 2000, A\&A, 362, 151

Enßlin, T. A., Biermann, P. L., Kronberg, P. P., et al. 1997, ApJ, 477, 560

Enßlin, T. A. 2002, A\&A, 396, L17

Feretti, L., Dallacasa, D., Giovannini, G., \& Tagliani, A. 1995, A\&A, 302, 680

Fujita, Y., Takizawa, M., \& Sarazin, C. L. 2003, ApJ, 584, 190
Fusco-Femiano, R., Orlandini, M., Brunetti, G., et al. 2004, ApJ, 602, L73

Gabici, S., \& Blasi, P. 2003a, Astropart. Phys., 19, 679

Gabici, S., \& Blasi, P. 2003b, ApJ, 583, 695

Gabici, S., \& Blasi, P. 2004, Astropart. Phys., 20, 579

Giovannini, G., Feretti, L., Venturi, T., et al. 1993, ApJ, 406, 399

Goldoni, P., Goldwurm, A., Laurent, P., et al. 2001, ESA SP-459, 165

Jaffe, W. J. 1977, ApJ, 212, 1

Kaastra, J. S., Lieu, R., Tamura, T., Paerels, F. B. S., \& den Herder, J. W. 2003, A\&A, 397, 445

Kim, K.-T., Kronberg, P. P., Dewdney, P. E., \& Landecker, T. L. 1990, ApJ, 355, 29

Kuo, P.-H., Hwang, C.-Y., \& Ip, W.-H. 2004, ApJ, 604, 108

Lieu, R., Ip, W.-H., Axford, W. I., \& Bonamente, M. 1999, ApJ, 510, L25

Miniati, F., Ryu, D., Kang, H., \& Jones, T. W. 2001a, ApJ, 559, 59

Miniati, F., Jones, T. W., Kang, H., \& Ryu, D. 2001b, ApJ, 562, 233

Miniati, F. 2002, MNRAS, 337, 199

Miniati, F. 2003, MNRAS, 342, 1009

Petrosian, V. 2001, ApJ, 557, 560

Pfrommer, C., \& Enßlin, T. A. 2004, A\&A, 413, 17

Reimer, O., Pohl, M., Sreekumar, P., et al. 2003, ApJ, 588, 155

Rephaeli, Y. 1979, ApJ, 227, 364

Rephaeli, Y., Ulmer, M., \& Gruber, D. 1994, ApJ, 429, 554

Rephaeli, Y., Gruber, D., \& Blanco, P. 1999, ApJ, 511, L21

Roland, J. 1981, A\&A, 93, 407

Sarazin, C. L. 1999a, ApJ, 520, 529

Sarazin, C. L. 1999b, MPE Rep., 271, 185

Schlickeiser, R., Rievers, A., \& Thiemann, H. 1987, A\&A, 182, 21

Struble, M. F., \& Rood, H. J. 1991, ApJS, 77, 363

Takizawa, M., \& Naito, T. 2000, ApJ, 535, 586

Thierbach, M., Klein, U., \& Wielebinski, R. 2003, A\&A, 397, 53

Venturi, T., Giovannini, G., \& Feretti, L. 1990, AJ, 99, 1381

Völk, H. J., Aharonian, F. A., \& Breitschwerdt, D. 1996, Space Sci. Rev., 75, 279

Weekes, T. C., Badran, H., Biller, S. D., et al. 2002, Astropart. Phys., 17,221 